\title{
Communication Technologies Enabling Effective UAV Networks: a Standards Perspective
}

\author{
Anna Maria Vegni, Senior Member, IEEE, Valeria Loscrí, Senior Member, IEEE, \\ Carlos T. Calafate, and Pietro Manzoni, Senior Member, IEEE
}

\begin{abstract}
Recent developments in the Unmanned Aerial Vehicles (UAVs) field have put in evidence the need for a standardization process of the communication technologies supporting direct information exchange, thus enabling UAV-to-UAV networking. We consider this is necessary to achieve all sorts of cooperative tasks requiring real-time (or near-real-time) synchronization, including swarm formation and collision avoidance. In this paper, we therefore argue in favor of introducing a new standard that would address this specific area, highlighting why current technologies are not adequate, what are the different steps towards a rapid standardization, and which lessons have been learned from related fields, namely the vehicular and the robotic environments, in the past few years.
\end{abstract}

\section{INTRODUCTION}

The Unmanned Aerial Vehicles (UAVs) sector has been experiencing an unprecedented growth in recent years, and was estimated to generate a total of 22.5 billion dollars at the end of 2020. Such estimations are even more promising in coming years, where a growth rate of $13.8 \%$ is foreseen, reaching 42.8 billion dollars by 2025 . This outstanding growth is quite expectable when considering the ever-growing number of application areas where the contribution of UAVs is significant, including site surveys, industrial inspection, surveillance, smart agriculture, search and rescue (SAR) missions, topography, parcel delivery, human transportation, and a large et cetera [1].

Recently, the coordination among UAVs is becoming critical in new emerging applications, such as UAV swarms, UAV collision avoidance systems, and cooperative UAV applications [2]. However, such new applications have a critical requirement in common, which is the need for a communications system that allows UAVs to directly connect to each other for data exchange. The main issue hindering such requirement is that, currently, commercial UAVs are equipped with no specific communication interface that enables UAV-to-UAV communications, as no technology has been yet standardized for this purpose. The IEEE, with the IEEE P1920.1 and IEEE P1920.2 Standards Working Groups, is addressing this goal, being P1920.1 dedicated to Aerial Communications and Networking Standards, and P1920.2 dedicated to developing

Manuscript received xxx

C. Calafate and P. Manzoni are with the Department of Computer Engineering, Universitat Politècnica de València, Valencia, Spain, 46022. E-mail: (calafate@disca.upv.es, pmanzoni@disca.upv.es).

A. M. Vegni is with the Department of Engineering, Roma Tre University, Italy. E-mail: annamaria.vegni@uniroma3.it

V. Loscrí is with the FUN Team at Inria Lille - Nord Europe, France. E-mail: valeria.loscri@inria.fr

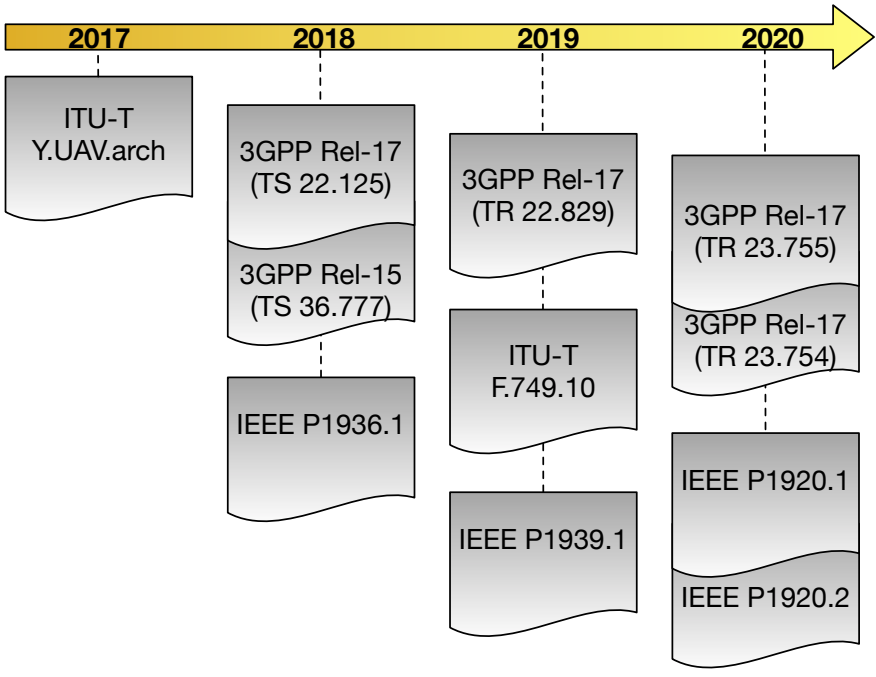

Fig. 1: Overview of main initiatives of UAV standardization.

a standard for Vehicle-to-Vehicle Communications for Unmanned Aircraft Systems. While the former defines air-to-air communications for self-organized ad-hoc aerial networks, the latter defines the protocol for exchanging information among vehicles.

The Third Generation Partnership Project (3GPP) is actively working in identifying the requirements, technologies and protocols for UAV communications, too. Initially, 3GPP TR 36.777 investigated how the enhanced long-term evolution can be employed to optimize UAV communications performance. Then, in 3GPP TS 22.125, the requirements for providing UAV services through 3GPP networks were defined, and in 3GPP TR 22.829 several UAV-enabled applications and use cases were described, to be supported by $5 \mathrm{G}$ networks. Finally, 3GPP TR 23.754 has focused on the network infrastructure and procedures to support the connectivity, identification, and tracking of UAVs, while 3GPP TR 23.755 also defined the application architecture to support efficient UAV operations. In a similar way, the ITU-T aligned with the $3 \mathrm{GPP}$ vision to establish a functional architecture for UAVs and remote controllers (RCs) accessing IMT-2020 networks. Fig. 1 provides a general overview of main initiatives towards a UAV communication standard, which are still ongoing.

The previous standardization activities focus on cellular technology as an enabling technology for communications, and do not address UAV-to-UAV communications. In this paper we present the points in favor and the need of starting a 
standardization process that would address this specific area, highlighting why current technologies are not adequate, what are the different steps towards a rapid standardization, and which lessons have been learned from related fields, like the vehicular and robotic environments, in the past few years. We think that providing a clear reference on the pending issues, taking as a reference what has been done in other fields, can help researchers in better taking steps toward the definition of adequate solutions, which are yet to be provided, tested and standardized in this field.

This paper does not aim to present a novel standard for UAV networking, which is a long and elaborated process, but rather to extract the experience and the lessons learned from fields like Intelligent Transportation Systems (ITS) and the robotics area, which have similar features and challenges as UAVs, and take advantage of the results available in those fields to address the area we are studying. This approach can help us at defining the issues and the requirements of UAV-to-UAV networking.

The remainder of this paper is organized as follows. In Section [II, we provide an overview of the different communication paradigms that are actually applicable to aerial vehicular environments. Section III presents an overview of different wireless technologies that can be adopted in aerial vehicular networks for communication purposes. Sections IV and V highlight the main lessons learned from the ITS and robotic area, respectively, and how such information can be adopted into the UAV domain. A final discussion is then included in Section VI, while conclusions are drawn at the end of the paper.

\section{OVERVIEW OF DIFFERENT COMMUNICATION PARADIGMS}

Multiple UAVs connected in wireless ad-hoc manner form a so called Flying Ad hoc Network (FANET), offering a multihop connectivity service to each other. UAVs can be equipped with different networking technologies for communication with multiple UAVs, and between UAVs and other systems such as the Ground Control Stations (GCS), Wireless Sensor Networks (WSN), and on-ground robots.

Specifically, in a FANET [3], [4], we can distinguish two main types of communication paradigms, i.e., (i) UAV-to-UAV (U2U) communications, where UAVs communicate with each other via Line-of-Sight (LoS) propagation links in a multihop manner in order to extend the communications range, and (ii) UAV-to-GCS communications (U2GCS), where a UAV communicates directly with the GCS. Mixing such two communication paradigms is the basis of hybrid approaches, which represent a combination between U2U and U2GCS communications, and where the UAV can send its data directly to the GCS in a one-hop or in a multi-hop fashion via the different UAVs in the mission area [5]. UAVs can then act as relay nodes for data forwarding in a FANET.

Fig. 2 depicts the main communication paradigms i.e., U2U and U2GCS, and vice versa. UAV capabilities and equipment can vary greatly depending on the specific UAV swarm application, and associated requirements. UAVs are expected to be largely integrated into existing wireless technologies provided by cellular and wireless networks, as well as satellite technology. UAV swarms allow connectivity among UAVs, and from UAVs to the ground, as well as from UAVs to satellites. As can be expected, UAVs shall be equipped with different network interface cards for technological heterogeneity needs, and will also require a specific communication standard.

\section{A. UAV-to-Ground Control Station communications (U2GCS)}

In this communication paradigm, UAV s can be connected either to terrestrial cellular or to satellite networks, with the aim of providing network connections for massive UAVs, while serving ground users [6]. Often, U2GCS communications are also called "network-connected UAV communications", where UAVs are integrated into the cellular network, depending on the role that UAVs play. Specifically, in cellular-enabled UAV communications, UAVs with their own missions (e.g., cargo delivery, video surveillance, video streaming, and so on) act as new aerial users, communicating with the cellular network through the existing ground base stations [7]. On the other hand, in UAV-assisted cellular communications, UAVs are connected to the cellular networks through the aerial relays, acting as gateways that perform direct U2GCS communications, and vice versa [8].

In general, U2GCS communication mode allows UAVs to communicate with the fixed central control center, such as a ground station, and are deployed to provide wireless services in case of terrestrial base station failure. U2GCS connectivity can be exploited to fill the gap between ground networks and satellites, thus representing an intermediate layer. In this scenario, UAVs can be exploited to cover connectivity holes, provide services in harsh environments, enhance network performance, etc.

Direct U2GCS communications exploit the unlicensed spectrum or reuse the spectrum bands that have been assigned for other particular applications, thus resulting in limited data rates, unreliable connections, and insecure communications. Other issues are related to signal propagation in free space, since high mobility of UAVs cannot always guarantee LoS propagation [9]. In such scenarios, large-scale fading occurs, as well as multiple reflections, scattering, and diffractions due to obstacles or unexpected strong interference (i.e., jamming signals). Furthermore, considering the high mobility of UAVs and the coverage requirements of swarm applications, the U2GCS mode is not stable, with the possibility that the entire communication network becomes paralyzed once the ground station or the satellite breaks down, loses the radio link, or is attacked.

\section{B. UAV-to-UAV communications ( $U 2 U)$}

Collaborative UAV fleets are expected to be able to communicate with each other using U2U mode. Each UAV in a swarm can establish efficient communications, both directly between two UAVs, or via multi-hop communication paths with other UAVs.

U2U communication mode shows similar features to ground device-to-device (D2D) communications including synchronization, peer discovery, mode selection, resource allocation, 


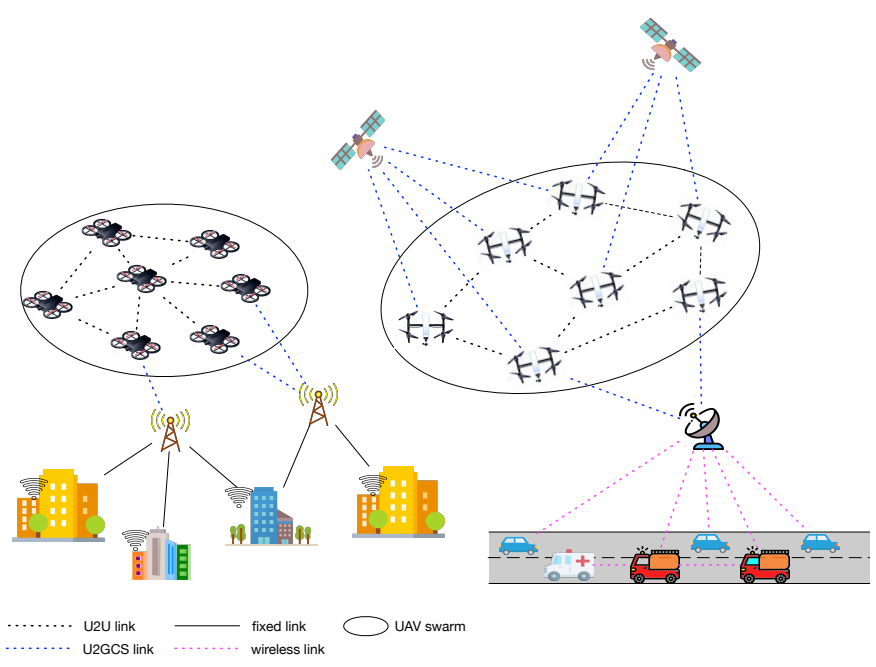

Fig. 2: UAV main communication paradigms, i.e. U2U and U2GCS, included in a typical example for aerial surveillance and imaging (left), and SAR applications (right). All the links are bidirectional.

interference management, security, and where the choice of the appropriate networking protocol depends on the nature of the application and the technology used. For instance, Bluetooth devices are mainly used for commercial/customer UAVs as a $\mathrm{RC}$, while WiFi technology is adopted for both remote control and video streaming applications, and $3 \mathrm{G} / 4 \mathrm{G}$ technology is used mostly for U2GCS. Also, specific parameters vary in case of different applications, such as the number and type of UAVs in the swarm, the on-board processing capabilities, memory and storage capacity, energy and power capability of the UAVs, transmission robustness, throughput and delay, and the UAV mobility. However, there may exist common requirements for all the different applications, such as lowenergy communications.

Finally, another important factor to account for is the size and weight of the hardware (HW) associated to the implementation of a specific communication, as many UAVs have small size and lifting capabilities. Hence, a standard should account for all the common requirements. Also, the choice of a specific UAV affects the operation range and the number of required UAVs. For instance, large UAVs with specialized radio transceivers can provide a longer range of connectivity over a single link. In contrast, commercial open source devices can provide the same range of connectivity via multi-hop propagation. Notice that the possible limitations of current HW do not prevent the definition of a whole-stack standard. HW could be a limitation depending on the complexity of the application running on the UAV, but this is outside the scope of this paper.

To summarize, in U2U communications there are a lot of variables that must be taken into account for an effective data communication system. In this regard, it is paramount to define a communication standard that accounts for all such variable parameters, and that can be suitable for different application scenarios.

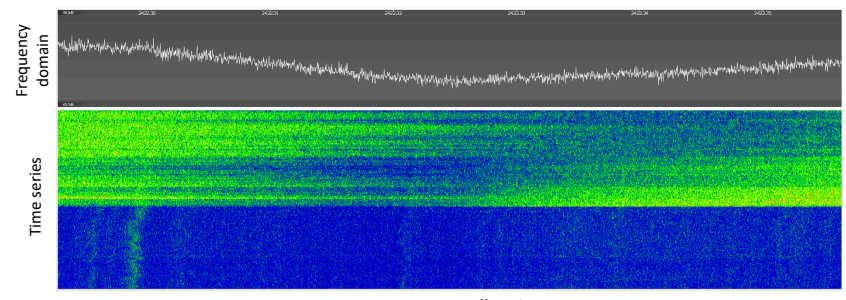

Remote controller ON

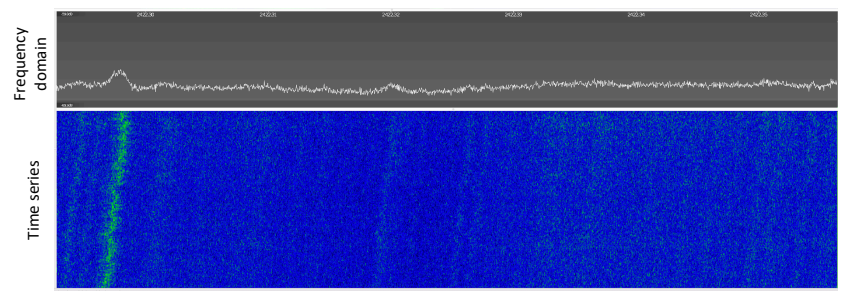

Remote controller OFF

Fig. 3: Spectral analysis of the impact of turning on the UAV RC for a common commercial device (FrSky Taranis X9D 2.4G ACCST). Center frequency: 2422.26 MHz.

\section{FEASIBILITY ANALYSIS OF DIFFERENT TECHNOLOGIES FOR U2U COMMUNICATIONS}

Communications among UAVs are necessary mainly to disseminate measurements, share tasks, and control information, as well as to coordinate the UAVs more effectively toward a global goal, such as monitoring a given area or detecting events in the shortest time possible, in disaster recovery situations.

UAVs pose some very strong constraints regarding communications, basically due to $(i)$ their possible high mobility, (ii) the inter-distance of devices that can easily reach kilometers, and that causes power attenuation (fading), (iii) the antenna positioning, which is key for link establishment, and, most importantly, (iv) the scarce energy available. The used wireless technologies must therefore adapt to such constraints and provide energy-efficient and low complexity communication service for a variety of uses that should be deployed on scalable networks.

In addition to these issues, most small UAVs are radiocontrolled, meaning that the RC must be available as backup even for mission-based flights. Such radio links involve an additional spectral occupancy, that introduces additional restrictions. For example, Fig 3 shows the spectral analysis for a FrSky Taranis RC, well known for its frequency hopping strategy denoted as Advanced Continuous Channel Shifting Technology (ACCST). We can observe that, when activating this RC, the frequency band is greatly disrupted, as can be seen both in the time series and in the snapshot of the frequency domain. In particular, we find a variable but wide occupation of the entire $2.4 \mathrm{GHz}$ band, which is associated to the frequency hopping strategy adopted, and that affects other transmissions in this free band.

When looking towards commercial products, there are already various available wireless data technologies. From the sensor network standards world, we could adopt technologies like ZigBee, RFID, or Bluetooth, that work over relatively short distances, up to a few hundred meters at most, with low 


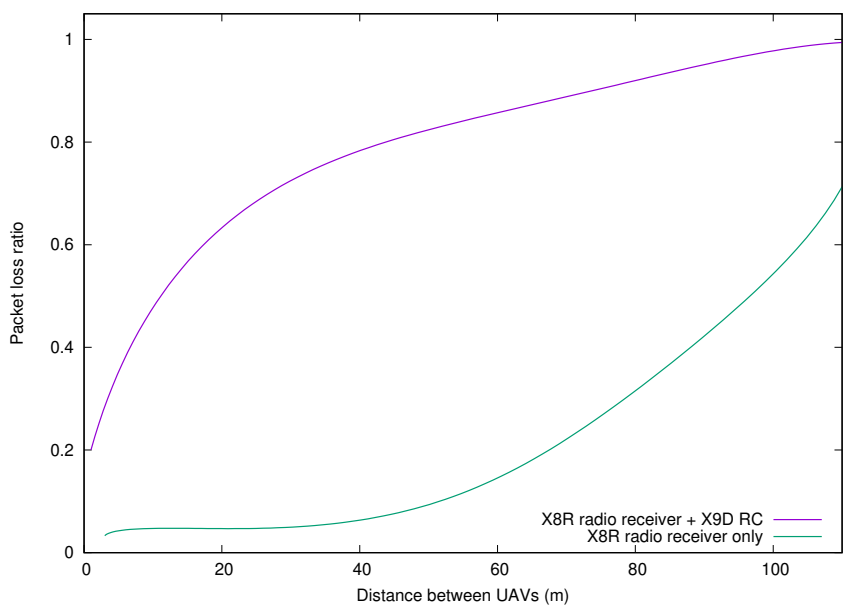

Fig. 4: Impact of RC technologies on U2U performance when using $\mathrm{WiFi}$ in the $2.4 \mathrm{GHz}$ band (only broadcast data at $6 \mathrm{Mbit} / \mathrm{s})$.

data rates and low energy consumption. Standards like GPRS, LTE, WiMAX, etc., work over long distances, and provide a high throughput, but they consume more energy, and demand for an expensive and fixed infrastructure of base stations, with an adequate link to the underlying network backbone.

Wi-Fi technology, based on the IEEE 802.11 standard, has continuously improved during the past years, where each generation had faster speeds and lower latency than the previous one, with a variety of device types. Currently used technologies include IEEE 802.11n, IEEE 802.11ac, and IEEE $802.11 a x$. The high availability of devices and drivers, the relatively long range under proper conditions, and the high bandwidth they offer, would make this technology the first immediate choice. Yet, when adopted for UAV environments, the RC interference issues depicted in Fig. 3 were found to have a very high impact in Wi-Fi performance based on real testbed experiments, as highlighted in Fig. 4. It is shown that, for a fixed inter-UAV distance, the packet loss ratio suffers of an increase in case of having the $\mathrm{RC}$ turned on (purple line), compared to the scenario with no RC available, but having the receiver device remain on (green line).

Another disadvantage is the energy cost of this technology, that would reduce the overall duration of the UAV batteries. The latest IEEE 802.11ah standard focuses on this limitation up to a certain extent by combining the advantages of Wi-Fi and low-power sensor network communication technologies, achieving communication over longer distances among a large number of low-power devices. IEEE 802.11ah is a wireless networking protocol published in 2016 as an amendment to the IEEE Std 802.11-2007 (which was a revision of IEEE Std 802.11-1999). It uses $900 \mathrm{MHz}$ license-exempt bands to provide extended range compared to conventional $\mathrm{Wi}-\mathrm{Fi}$ networks operating at $2.4 \mathrm{GHz}$ and $5 \mathrm{GHz}$. It also benefits from lower energy consumption, allowing the creation of large groups of stations or sensors that cooperate to share signals. Thanks to its low power consumption, it is a good competitor of Bluetooth, and has the added benefit of offering higher data rates and wider coverage range. Unfortunately, commercial
IEEE 802.11ah chipsets remain unavailable, and their possible adoption in UAV field will take time.

Another good candidate is the Bluetooth Low Energy (LE) technology. It was introduced in the Bluetooth 4.1 specification from the Bluetooth Special Interest Group, and requires much less energy, i.e., slave nodes can work for months on a single coin cell. Bluetooth LE is interesting for communication among UAVs for various reasons. The radio transmission is designed to spend nearly all of the time in the power saving mode, thus requiring very low power. Radio range is close to 100 meters, and the Bluetooth LE radio is highly resistant to interference when using the $2.4 \mathrm{GHz}$ band. Regarding pure data transfers, this technology is very fast, i.e., the whole process that involves the establishment of a connection between a node and a master device, the data transferal, and the connection shut down, can take place in just $3 \mathrm{~ms}$. Finally, Bluetooth is so widely known and used that its availability and usability is extremely wide.

Compared with ZigBee and $\mathrm{Wi}-\mathrm{Fi}$, the former provides a flexible mesh networking architecture, ideal for complex and multi-node networks, and the latter clearly supports higher data rates. Nevertheless, Bluetooth LE surpasses both technologies thanks to its low cost, simplicity and ultra-low power consumption when transmitting small amounts of data. This last aspect is very relevant, as Bluetooth LE does not offer classical Bluetooth-style capabilities in a more energy-efficient form. If, for communication among UAVs, we need classic Bluetooth functionalities like streaming of audio or other data transfers supporting data rates of $1 \mathrm{Mbps}$ or higher, we will still have the same high power budget. Bluetooth LE is useful to quickly establish a connection, transmit short bursts of tiny packets of data, and then quickly disconnect. Such restriction, along with the limited radio range, may fail to serve the purposes of UAV communications in different cases.

Last, the surge of the LPWAN technologies, like SIGFOX, NB-IoT and, in particular, LoRa, can open-up new possibilities for UAV interconnection. LoRa uses license-free sub-gigahertz radio frequency bands like $433 \mathrm{MHz}, 868 \mathrm{MHz}$ (in Europe), and $915 \mathrm{MHz}$ (in Australia and North America). It enables long-range transmissions up to various kilometers with low power consumption, achieving data rates from $0.3 \mathrm{kbps}$ to $27 \mathrm{kbps}$ depending upon the different modulation configurations. On the negative side, LoRa has a very simple MAC protocol that would not make it adequate for reliable transmissions without a proper transport layer protocol to make it more robust, but its physical layer characteristic and its low power requirements make it really interesting. Nevertheless, the slow transmission rates offered fail to meet the lowdelay and throughput requirements of many UAV applications. Table I] collects different metrics expressed in terms of latency, bandwidth, radio coverage, power usage, robustness and technology maturity, which allow to identify some potential technology candidate for $\mathrm{U} 2 \mathrm{U}$ communications.

\section{LESSONS LEARNT FROM THE ITS AREA}

In recent years, the ITS field has witnessed significant improvements of the available wireless communication technologies. An example of such a technology is detailed in 
TABLE I: Summary of the characteristics of four candidate technologies for U2U communications. Robustness refers to the required SNR for a BER of $10^{-4}$.

\begin{tabular}{|c|c|c|c|c|c|c|c|}
\hline Technology & $\begin{array}{l}\text { Radio } \\
\text { range }\end{array}$ & $\begin{array}{c}\text { Power } \\
\text { usage }\end{array}$ & $\begin{array}{l}\text { Radio } \\
\text { band }\end{array}$ & Bandwidth & Latency & Robustness & $\begin{array}{l}\text { Technology } \\
\text { Maturity } \\
\text { (TRL) }\end{array}$ \\
\hline Wi-Fi & Few km & High & $\begin{array}{c}2.4 / 5.0 \\
\mathrm{GHz}\end{array}$ & Very high & $\begin{array}{l}50 \mathrm{~ms} \\
\text { High }\end{array}$ & $\begin{array}{c}15.2 \text { to } 17 \\
\text { dB } \\
\text { (Medium) }\end{array}$ & 9, High \\
\hline Bluetooth & $100 \mathrm{~m}$ & $\begin{array}{c}\text { Moderate } \\
\text { to very } \\
\text { low }\end{array}$ & $2.4 \mathrm{GHz}$ & $\begin{array}{c}\text { Moderate } \\
\text { to low }\end{array}$ & $\begin{array}{l}100 m s \\
\text { High }\end{array}$ & $\begin{array}{c}9.5 \text { to } 14 \\
\text { dB } \\
\text { (Medium) }\end{array}$ & 9, High \\
\hline LoRa & Several km & Very low & sub GHz & Low & $\begin{array}{c}\text { Low } \\
\text { Latency } \\
\text { Class-C }\end{array}$ & $\begin{array}{c}14 \mathrm{~dB} \\
\text { (Medium) }\end{array}$ & 9, High \\
\hline $\begin{array}{c}\text { IEEE } \\
802.11 a h\end{array}$ & Few km & $\begin{array}{c}\text { Moderate } \\
\text { to very } \\
\text { low }\end{array}$ & $900 \mathrm{MHz}$ & Low & $\begin{array}{c}50 \text { to } \\
100 \mathrm{~ms} \\
\text { High }\end{array}$ & $\begin{array}{c}15 \mathrm{~dB} \\
\text { (Medium) }\end{array}$ & 6, Medium \\
\hline
\end{tabular}

the $802.11 p$ annex of the IEEE 802.11 standard [10], which regulates communications in the $5.9 \mathrm{GHz}$ band for vehicleto-vehicle (V2V) and vehicle-to-infrastructure (V2I) wireless networking. This technology was specifically designed for vehicular environments, including crash-prevention solutions and alerts in general, platooning, and all types of safety and infotainment support [11]. It benefits from a reserved band of frequencies, which constitute a few of the key reasons why both its applicability and performance have proved to achieve very high levels. Nevertheless, a very slow standardization and adoption, along with the introduction of $5 \mathrm{G}$ technologies, has lessened the adoption of IEEE 802.11p as the technological choice for vehicular environments.

When analyzing the different requirements for deploying a wireless communications technology in UAV environments, such that this technology becomes an enabler of both $\mathrm{U} 2 \mathrm{U}$ and U2GCS communication modes, we find great resemblances with vehicular environments, where both V2V and V2I communications must be supported. In particular, different features of IEEE $802.11 p$ standard would be applicable and highly recommended to UAV environments. Among such features, we remind:

- Reduced channel width: by adopting a narrow channel width of $10 \mathrm{MHz}$ instead of the $20 \mathrm{MHz}$ (or more) defined for wireless LAN uses of the standard, the technology is able to better cope with Doppler effects associated to high levels of mobility, which are typical of both vehicular and UAV networks;

- Operation Outside the context of a Basic Service Set (BSS): in plain IEEE 802.11 all frames include a BSSID field containing relevant data, such as the MAC address of an access point when using infrastructure, or a randomly generated MAC address when creating an ad-hoc network. Hence, ad-hoc networks created in different places will not communicate with each other, which is a big issue hindering vehicular communications. Similarly, operations with UAVs requiring interoperability for communications and maximum flexibility cannot be constrained by BSSID or SSID issues appearing in standard IEEE 802.11 networks, and must drop their functionality similarly to what was done when targeting ground vehicle environments;
- Assignment of reserved channels: as discussed earlier, the $2.4 \mathrm{GHz}$ ISM band is massively used by UAVs when radio RCs are active, which hinders the use of this particular band for any other purposes. The $5.8 \mathrm{GHz}$ band is a better alternative, although in urban environments we see that it is intensively used by home users to create Wi-Fi hotspots. Neither of these bands was created specifically for UAV communication purposes, meaning that many unexpected competing technologies can adhere to these bands at any time and make channel occupation unfeasible for a correct operation in the target UAV area. So, similarly to what was done for ground vehicle environments, a narrow channel space should be reserved to specifically fill-in for the needs of aerial vehicle communications in the civilian and industrial sectors (as the military sector already benefits from other reserved channels).

Finally, speed is actually a critical factor in the ITS and UAV worlds. This is why we believe that the experience coming from the ITS area, where relative speeds between vehicles can reach $260 / 300 \mathrm{~km} / \mathrm{h}$, is also significant in this context.

Despite the similarities between UAVs and ground vehicles, there are also several differences that impose technological restrictions from a communications perspective. For instance, UAVs are characterized by being endowed with batteries with a relatively low size and weight, meaning that the power consumption associated to wireless communications can become more of an issue compared to the ground vehicles counterpart. For this reason, the number of wireless interfaces should be kept to a minimum (just one), which differs from vehicular environments where different interfaces may be operating in different channels, adopting different functions, as we can find one control channel $(\mathrm{CCH})$ and several service channels ( $\mathrm{SCH})$.

Furthermore, the size of the antennas adopted must be kept small -only a few centimeters at most- for a balanced installation and fitting in the UAV itself, especially when targeting UAVs of less than $4 \mathrm{~kg}$. Hence, overall, UAV networking is characterized by fewer requirements in terms of number of channels and services, which match their lower capabilities in terms of load capacity and power consumption. 


\section{LESSONS LEARNT FROM THE ROBOTICS AREA}

Often, UAVs are also referred to as unmanned aerial robots, due to some common similarities to the robotic area. Robots are typically organized in swarms with communication capabilities i.e., (i) between a user and the networked swarm, and (ii) intra-swarm communications, where each robot communicates with its neighbors. They may be equipped with multiple wireless technologies that can be used for different types of communications. For short range communication, a robot swarm can rely on Bluetooth, while using Wi-Fi for communications over relatively larger distances.

For such reasons, with the aim of pushing UAV research and standardization activities towards such communication technologies, we can learn from what UAVs have in common with "traditional" ground robots from a communication perspective. In this sense, we regard the modus operandi of the National Institute of Standard and Technology (NIST) for pushing standard development of wireless robotics communication [12]. The Department of Homeland Security (DHA) Science and Technology (S\&T) Directorate, together with NIST, started to regard to the specific requirements in terms of performance requirements. Even though the first results of this study were published in 2007, some conclusions are incredibly present-day and applicable to UAV wireless communication paradigms. Indeed, the authors of the study conclude that for successful and high performance communication, there is a need for a many-facet approach. In addition, new access schemes and Software-Defined Radio for interoperable communication schemes should be considered.

In a similar direction, authors of [13] propose simple but yet realistic wireless link models that remained unavailable for networked robotics, despite the literature is rich in wireless models for cellular applications. In particular, they consider experimental results for validating the wireless channel models for robotic networked systems. They argue that the specific environment needs an ad-hoc study for a correct capture of the key features to be considered in a wireless communication paradigm. Nevertheless, even though we agree that UAVs are a special type of robot, the specific environment (i.e., free space) where the communication occurs is pretty different from the one of ground robots. On the other hand, the way the authors proceeded in [13] suggests that, for a real advancement in wireless communication paradigms for UAVs, there is a need of collecting real measurements from the environment where the nodes operate, in order to identify the specific features to integrate in realistic link models.

Finally, very interesting is the discussion in [14], where Schiøler and Toftegaard perform a study for creating a standard for wireless robotic communication. They find that standardization efforts in this specific field needs to account for the immense diversity of the applications areas, and then the standardization across the entire set of applications is simply non productive and yet unfeasible. We believe that similar conclusions are also valid for the UAV wireless communications, and that few specific features that are common to the different applications areas need to be efficiently identified. One of the first attempt in this direction has been considered in [15].
TABLE II: Suggested steps prioritization for a rapid standardization of U2U communications.

\begin{tabular}{c|c}
\hline \hline Steps/Research Activities & Priority \\
\hline Channel Modeling & Very high \\
\hline Frequency Selection & Very high \\
\hline Modulation Schemes & High/Medium \\
\hline PHY/MAC Protocol Design & Medium \\
\hline Emerging Communication Technologies & Medium \\
\hline Full Networking Stack & Low \\
\hline \hline
\end{tabular}

In summary, we can conclude that despite there are several common points with the standardization efforts in the ground robotics wireless communication, some important differences need to be accounted for in the case of UAVs. In particular, the aerial environment of UAVs requires ad-hoc communication technology to address its specific features, as well as the definition of specific performance metrics like the UAV connectivity, or the energy consumption impact on the communication protocols.

\section{DISCUSSION}

In the previous sections we have highlighted the fact that $\mathrm{U} 2 \mathrm{U}$ communication remains as an open issue due to the lack of a specific technology for support, and since existing technologies do not fully meet the requirements of such environments. But what are the practical implications of such technological shortage?

Nowadays we can basically find two types of UAVs, i.e. (i) off-the-shelf UAVs, that are usually equipped with closed flight controllers, and (ii) custom UAVs, which may be equipped with either open or closed flight controllers, although only open controllers allow for novel research solutions to be developed. Anyway, in both cases, we find that this lack of standardization in the UAV sector leads to a lack of available communication systems integrated in these flight controllers, being only a very limited set of options available (e.g. $100 \mathrm{~mW}$ Radio Telemetry Transceivers for the $433 \mathrm{MHz}$ band). Hence, more sophisticated communication systems for either U2U or U2GCS support must make use of ad-hoc extensions that rely on additional embedded HW, and the chosen wireless networking interfaces. In addition, since such extensions will be very customized and do not benefit from a widespread vendor support, there are no guarantees of interoperability between devices, a requirement that is critical for applications such as collision avoidance during flight between UAVs of different vendors/owners.

To address the aforementioned problem, we consider that only a strong and fast standardization process, involving experts from both academia and industry, can provide an effective solution that benefits from a widespread adoption at a global scale. Without it, only limited solutions can be provided in research environments, and possible commercial products, if any, will be characterized by incompatibilities and vendor lock-in issues. In Table III we summarize some fundamental steps in order to accelerate the standardization process of aerial communication networks.

Leveraging on early results from ITS and the robotics area, which have issues similar to UAVs, as a basis for such a 
standardization process, we firstly consider the investigation of lower protocol layers (requiring high priority), and then the higher ones (requiring medium and low priority). Specifically, we have identified two main phases with high priority, i.e. (i) channel modeling and (ii) frequency selection, since they could account for the specificity of the environment where the drones communicate. The channel modeling allows to investigate the signal propagation in LoS in free space, subjected to noise and interference due to weather conditions. Having a dedicated frequency could also improve the interference mitigation with other existing technologies. Based on those most important steps, it will be possible, on the medium term, to conceive ad-hoc modulation schemes and a PHY/MAC protocol design with a low impact on energy consumption, which is one of the most important aspects to consider for $\mathrm{U} 2 \mathrm{U}$ communications. Emerging communication technologies can be accounted for in this process, as such requirements are shared with other research areas having common challenges, and hence synergies are possible. Finally, on a longer term, it will be desirable to have a full networking stack able to provide routing, security, management (including swarm management), and application layer functionalities.

To make such fast standardization possible, we refer to the IEEE 802.11 standard, and its " $p$ " annex for vehicular environments, which sets a good ground for the UAV sector as well, as many of the technical aspects of ground vehicular networks are equally applicable to aerial vehicular networks, and a new solution for the latter could be derived within a short time frame. Alternatively, a brand new standard that is designed from scratch, targeting solely vehicular aerial networks, could be developed. Such approach, despite involving a slower development process, has the potential of better meeting the target requirements of these aerial environments, thus achieving better performance in terms of Quality of Service by addressing the real-time and reliability constraints associated to critical applications, areas where IEEE 802.11related approaches typically under-perform.

Either way, both paths would lead to the ultimate and no longer postponable goal of introducing a standard that would meet the specific needs of aerial networks.

\section{CONCLUSIONS}

Currently, a fast growth of the UAV sector is happening due to the availability of adequate support systems, technologies and standards. Communication technologies are one of the key fields, being reliable, low delay and high-bandwidth communications a sine qua non condition for such advancement.

In this paper, we focused on communication systems for UAVs, highlighting the different communication modes and associated requirements, and analyzing different wireless communication technologies that may be applicable. We have highlighted the fact that, despite U2GCS communications are being successfully handled by different standards, U2U communication remains an open issue due to the lack of a specific technology for support, and since existing technologies do not fully meet the requirements of such environments.

Furthermore, we have argued in favor of introducing a new standard to specifically address U2U communications, highlighting the close resemblance with ground vehicle communications, and then the introduction of the " $p$ " annex of the IEEE 802.11 standard is expected to meet the specific requirements of those environments. We are strongly convinced that only by introducing a new standard for $\mathrm{U} 2 \mathrm{U}$ communications can the full potential of these flying devices be completely unleashed, and advanced applications like UAV swarms and crash avoidance protocols benefit from a widespread adoption.

\section{ACKNOWLEDGMENTS}

This work was partially supported by the "Ministerio de Ciencia, Innovación y Universidades, Programa Estatal de Investigación, Desarrollo e Innovación Orientada a los Retos de la Sociedad, Proyectos I+D+I 2018", Spain, under Grant RTI2018-096384-B-I00.

\section{REFERENCES}

[1] H. Shakhatreh, A. H. Sawalmeh, A. Al-Fuqaha, Z. Dou, E. Almaita, I. Khalil, N. S. Othman, A. Khreishah, and M. Guizani, "Unmanned Aerial Vehicles (UAVs): A Survey on Civil Applications and Key Research Challenges," IEEE Access, vol. 7, pp. 48 572-48 634, 2019.

[2] G. Chmaj and H. Selvaraj, "Distributed Processing Applications for UAV/drones: A Survey," in Progress in Systems Engineering, H. Selvaraj, D. Zydek, and G. Chmaj, Eds. Cham: Springer International Publishing, 2015, pp. 449-454.

[3] S. Zaidi, M. Atiquzzaman, and C. T. Calafate, "Internet of Flying Things (IoFT): A Survey," Computer Communications, vol. 165, pp. 53-74, 2021.

[4] J. Wang, C. Jiang, Z. Han, Y. Ren, R. G. Maunder, and L. Hanzo, "Taking drones to the next level: Cooperative distributed unmannedaerial-vehicular networks for small and mini drones," IEEE Vehicular Technology Magazine, vol. 12, no. 3, pp. 73-82, 2017.

[5] S. Zhang, H. Zhang, B. Di, and L. Song, "Cellular UAV-to-X Communications: Design and Optimization for Multi-UAV Networks," IEEE Transactions on Wireless Communications, vol. 18, no. 2, pp. 13461359, 2019.

[6] X. Chen, J. Tang, and S. Lao, "Review of Unmanned Aerial Vehicle Swarm Communication Architectures and Routing Protocols," Appl. Sci., vol. 10, no. 3661, 2020.

[7] S. Zhang, Y. Zeng, and R. Zhang, "Cellular-enabled UAV communication: A connectivity-constrained trajectory optimization perspective," IEEE Transactions on Communications, vol. 67, no. 3, pp. 2580-2604, 2019.

[8] H. Wang, J. Wang, J. Chen, Y. Gong, and G. Ding, "Networkconnected UAV communications: Potentials and challenges," China Communications, vol. 15 , no. 12, pp. 111-121, 2018.

[9] P. S. Bithas, V. Nikolaidis, A. G. Kanatas, and G. K. Karagiannidis, "Uav-to-ground communications: Channel modeling and uav selection," IEEE Transactions on Communications, vol. 68, no. 8, pp. 5135-5144, 2020.

[10] "IEEE Standard for Information technology_-Telecommunications and information exchange between systems Local and metropolitan area networks-Specific requirements - Part 11: Wireless LAN Medium Access Control (MAC) and Physical Layer (PHY) Specifications," IEEE Std 802.11-2016 (Revision of IEEE Std 802.11-2012), pp. 1-3534, 2016.

[11] G. Karagiannis, O. Altintas, E. Ekici, G. Heijenk, B. Jarupan, K. Lin, and T. Weil, "Vehicular networking: A survey and tutorial on requirements, architectures, challenges, standards and solutions," IEEE Communications Surveys Tutorials, vol. 13, no. 4, pp. 584-616, 2011.

[12] C. A. Remley, G. Koepke, E. Messina, A. Jacoff, and G. Hough, "Standards Development for Wireless Communications for Urban Search and Rescue Robots I NIST," 2007.

[13] A. Gonzalez-Ruiz, A. Ghaffarkhah, and Y. Mostofi, "A Comprehensive Overview and Characterization of Wireless Channels for Networked Robotic and Control Systems," Journal of Robotics, pp. 1-3534, 2011.

[14] H. Schiøler and T. S. Toftegaard, "Wireless Communication in Mobile Robotics a Case for Standardization," Wireless Personal Communications, vol. 64, pp. 583-596, 2012.

[15] H. Nawaz, H. M. Ali, and A. Laghari, "UAV Communication Networks Issues: A Review," Archives of Computational Methods in Engineering, pp. 1-21, 2020. 
Anna Maria Vegni is a tenure-track Assistant Professor in the Department of Engineering at Roma Tre University (Italy), since March 2020. She received the Ph.D. degree in Biomedical Engineering, Electromagnetics and Telecommunications from the Department of Applied Electronics at Roma Tre University, in March 2010. Her research activity focuses on wireless communications, vehicular networks and Internet of Things.

Valeria Loscrí is research scientist at Inria Lille - Nord Europe (France) in the FUN (Self-Organizing Future Ubiquitous Networks) team. She received the $\mathrm{Ph} . \mathrm{D}$. degree in Telecommunications and Electronics Systems from the University of Calabria in January 2007. Her research activities focus on wireless communication, non-conventional communication paradigms, cybersecurity in wireless networks and vehicular networks communications.
Carlos T. Calafate is a Full Professor in the Department of Computer Engineering at the "Universitat Politècnica de València (UPV)" in Spain. He graduated with honours in the University of Oporto (Portugal) in 2001. He received his Ph.D. degree in Informatics from the UPV in 2006. His research interests include ad-hoc and vehicular networks, UAVs, Smart Cities \& IoT. He is ranked among the top 100 Spanish researchers in the Computer Science \& Electronics field.

Pietro Manzoni is a Full Professor of Computer Networks at the "Universitat Politècnica de València (UPV)", Spain. He holds a Ph.D. from the "Politecnico di Milano", Italy. His research activity is focused on networking and mobile systems for the Internet of Things for Smart Cities and Rural Areas, LPWANbased networks, and Pub/Sub systems. He is a senior member of the IEEE. 